\title{
Effect of Hydrogen Bonds on the Dielectric Properties of Interfacial Water
}

\author{
Sleeba Varghese, ${ }^{\dagger}$ Sridhar Kumar Kannam, ${ }^{\ddagger}, \|$ Jesper Schmidt Hansen, ${ }^{\S}$ and Sarith P. Sathian ${ }^{*}{ }^{\dagger} \odot$ \\ ${ }^{\dagger}$ Department of Applied Mechanics, Indian Institute of Technology Madras, Chennai 600036, India \\ ${ }^{\ddagger}$ Faculty of Science, Engineering and Technology, Swinburne University of Technology, Melbourne, Victoria 3122, Australia \\ ${ }^{\S}$ Department of Science and Environment, Roskilde University, Roskilde 4000, Denmark \\ "School of Applied Sciences, RMIT University, Melbourne, Victoria 3001, Australia
}

ABSTRACT: The dielectric constant for water is reduced under confinement. Although this phenomenon is well known, the underlying physical mechanism for the reduction is still in debate. In this work, we investigate the effect of the orientation of hydrogen bonds on the dielectric properties of confined water using molecular dynamics simulations. We find a reduced rotational diffusion coefficient for water molecules close to the solid surface. The reduced rotational diffusion arises due to the hindered rotation away from the plane parallel to the channel walls. The suppressed rotation in turn affects the orientational polarization of water, leading to a low value for the dielectric constant at the interface. We attribute the constrained out-of-plane rotation to originate from a higher density of planar hydrogen bonds formed by the interfacial water molecules.

\section{INTRODUCTION}

Confinement induces significant changes in the structural and dynamical properties of water with most perturbations being extremely local and observable only within a few molecular layers close to the surface. ${ }^{1-3}$ However, recent studies indicate that the effect of confinement on the dielectric properties of water extends well beyond the interfacial regions. ${ }^{4,5}$ The extremely low dielectric constant of nanoconfined water also suggests a far more significant influence by the interfaces on the dielectric properties, when the confinement width reduces to nanometric scales. ${ }^{5-10}$ Despite these data available on the dielectric permittivity of water, knowledge on the physical origin of the anomalous dielectric behavior of confined water is still not fully complete. Understanding the underlying physical mechanism of the dielectric response is essential in the solvation dynamics of aqueous solutions ${ }^{11-13}$ that are primarily governed by the bulk dielectric properties of the solvent as well as in the development of nanoscale electromechanical devices. $^{14,15}$

Experimental investigations of the problem are very complicated. This has led to the development of theoretical and computer simulation techniques to calculate the molecular dielectric response in highly confined geometries. An appropriate theoretical model should at least be able to reproduce the experimental dielectric constant of homogeneous water. The Kirkwood-Fröhlich fluctuation formula ${ }^{16,17}$ has shown satisfactory results on the static dielectric constant of homogeneous water for different temperature ranges. ${ }^{18,19}$ However, this approach has significant limitations when

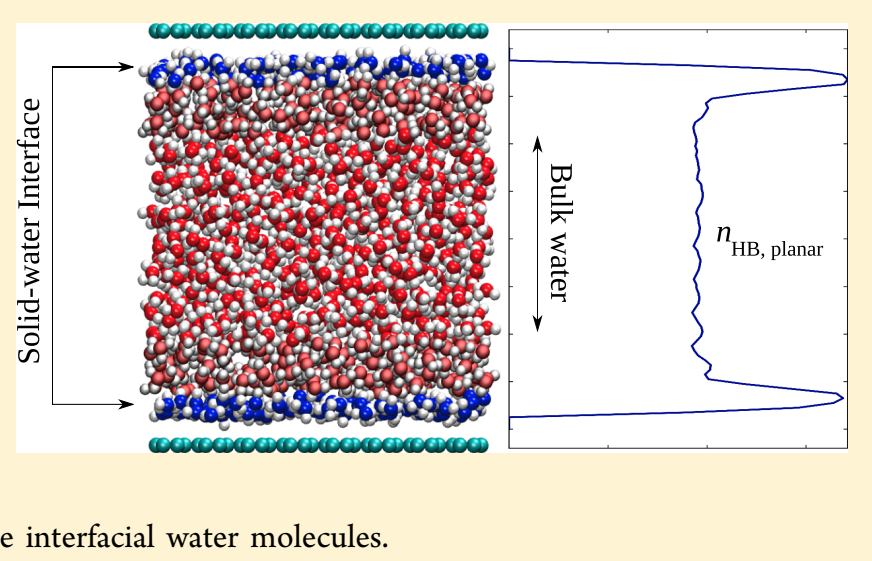

applied to confined systems. For instance, while calculating permittivity of an interfacial layer, the Kirkwood-Fröhlich fluctuation formula neglects the dipolar contribution of water molecules away from the layer but is significant due to the substantial ordering of water molecules near a solid surface. $^{20,21}$ Ballenegger and Hansen ${ }^{22}$ addressed these issues and derived a correct fluctuation formula to calculate the dielectric permittivity of confined polar fluids. They found that under confinement, the dielectric constant is a space-dependent dielectric response function with components parallel and perpendicular to the surface. Later, Bonthuis et al. ${ }^{23}$ modified the fluctuation formula to calculate the dielectric properties of molecules with higher multipole moments (e.g., water). Both studies observed the emergence of singularities in the perpendicular dielectric response function at the molecular layers close to the solid surface. Further investigations into the interfacial dielectric properties revealed the existence of an abnormally high polarization field close to the surface, which overscreens any external electric field. ${ }^{22,23}$ The consequence of this overscreening phenomenon is the negative values observed in the dielectric permittivity profile of water close to the solid surface, which ultimately leads to a low value of the effective dielectric constant in the interface regions. ${ }^{24}$

Experimental and simulation studies show a substantial decrement in the perpendicular component of dielectric

Received: February 24, 2019

Revised: $\quad$ May 22, 2019

Published: May 23, 2019 
(a)

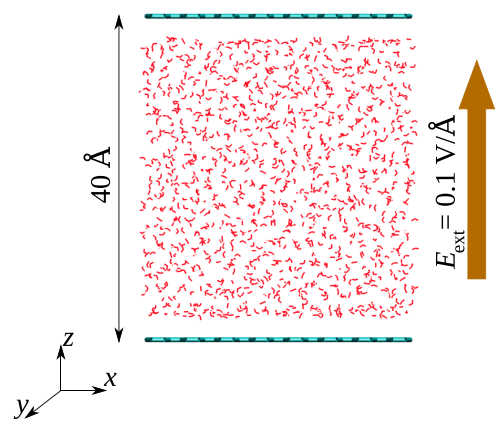

(b)

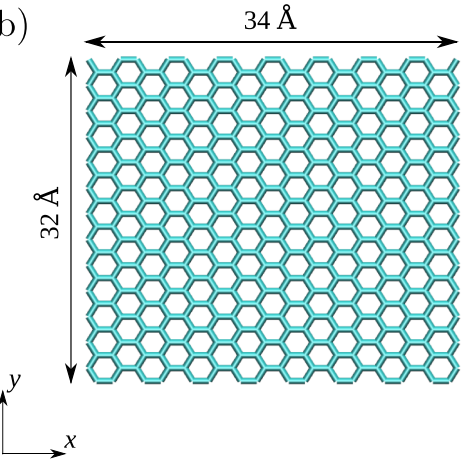

Figure 1. (a) Schematic representation of the confined water system (b) Top view of the graphene channel.

permittivity for a few layers of water molecules near the solid surface. $^{23-27}$ Even though this interfacial region with the reduced dielectric response extends only up to a few nanometers, under confinement, its influence on the overall dielectric permittivity becomes quite significant. Molecular dynamics simulation studies reported an extremely low effective dielectric constant for water under nanoconfined geometries. ${ }^{6,8} \mathrm{~A}$ recent experiment on the water confined between hexagonal boron nitride layers substantiated this low dielectric response under nanoconfinement and it was observed that water regains its bulk permittivity only when channel widths are higher than $100 \mathrm{~nm}$.

This reduced dielectric response under confinement is modeled on the basis of the capacitor model approach, which considers the overall permittivity as a series of low-permittivity interfacial regions and a nominal permittivity bulk region. ${ }^{9,28}$ Although the capacitor model explains the overall permittivity of a confined system, the physical origin of the reduced dielectric response is still to be pondered. Understanding the physical mechanism of this reduced dielectric response at the interfaces requires investigation into the distinctive structural features of water close to solid surfaces, which can be rationalized in terms of intermolecular hydrogen bonding of water. Hence, like most anomalous properties, any deviation in the dielectric behavior of water, we here conjecture, should also arise from the restructuring of its hydrogen-bond network. ${ }^{29-31}$ Despite the large amount of knowledge gathered, the influence of hydrogen bonds on the dielectric permittivity of water is still unclear. ${ }^{26,27,31,32}$

In this work, we investigate the orientation of hydrogen bonds and its correlation with the dielectric permittivity of confined water. We find a higher density of hydrogen bonds parallel to the walls at the solid-water interface when compared with the bulk liquid. This enhanced planar hydrogen bond network constrains the rotation of water molecules to the planes parallel to the channel walls. This extensive network of planar hydrogen bonds indicates that the reorientation of water molecules in the direction perpendicular to the channel walls is energetically unfavorable. The consequence will be low polarizability of the interfacial water molecules under an external electric field. The next section provides a detailed description of the molecular dynamics simulation methods employed, which is followed by a section on the Results and Discussion.

\section{METHODS}

We perform molecular dynamics simulations of a graphene nanochannel enclosing $\mathrm{SPC} / \mathrm{E}^{33}$ water using the large atomic/molecular massively parallel simulator ${ }^{34}$ package. The bond and angular vibrations of each water molecule are constrained using the SHAKE $^{35}$ algorithm. The SPC/E water model has a dipole moment of $2.35 \mathrm{D}$, in agreement with the experimental value of $2.9 \pm 0.6 \mathrm{D} .^{36}$ Graphene sheets are placed in the $x-y$ plane with periodicity in both directions, and $z$ is the perpendicular or confined direction, as shown in Figure 1. Liquid-surface chemical reactions and atomic and electronic polarizability effects are neglected. The graphene walls are kept as rigid, uncharged, and electrically transparent throughout the simulations. Each hydrogen atom carries a partial charge of 0.4238 e and oxygen atoms carry a partial charge of $-0.8476 e$, where $e$ is the elementary charge. The repulsive and attractive parts of van der Waals interaction are modeled using the pairwise additive Lennard-Jones (LJ) potential with a cutoff length of $10 \AA$. The van der Waals parameters for water-carbon (WC) interaction $\left(\epsilon_{\mathrm{WC}}=0.09369 \mathrm{kcal} /\right.$ mol, $\left.\sigma_{\mathrm{WC}}=3.19 \AA\right)$ are taken from Werder et al. ${ }^{37}$ The Lennard-Jones parameters for oxygen-oxygen $(\mathrm{OO})$ interaction are $\epsilon_{\mathrm{OO}}=0.15535$ $\mathrm{kcal} / \mathrm{mol}$ and $\sigma_{\mathrm{OO}}=3.166 \AA$, and Lennard-Jones interaction coefficients are zero for hydrogen atoms. The short-range Coulombic interactions with the charged sites are also modeled with the same truncation cutoff of $10 \AA$. Long-range electrostatic interactions are calculated using the Ewald algorithm with a particle-particleparticle-mesh solver ${ }^{38}$ of LAMMPS $^{34,39}$ with a relative root mean square error in the per-atom force calculations below $1 \times 10^{-4}$. Nonperiodicity in the $z$-direction is accommodated in the Ewald algorithm by inserting empty volumes above the channel walls such that the extended confined dimension is 3 times the actual channel size and a correction term in the calculation of long-range Coulombic forces. $^{34,39}$ The corrected Ewald algorithm is known as EW3DC. ${ }^{39}$ The simulation box dimensions for the graphene channel in the $x-y$ plane are $L_{x}=34 \AA$ and $L_{y}=32 \AA$. Channel width is kept at $40 \AA$. Water up to $5 \AA$ from the graphene surface ( $0-5$ and $35-40 \AA$, which includes the density peaks near the wall) is considered as interfacial water. ${ }^{3}$ The equilibrium density for water inside a nanochannel is obtained by immersing our graphene sheets inside a $50 \times 50 \times 60 \AA^{3}$ water reservoir at the ambient conditions of $1.01 \mathrm{bar}$ pressure and temperature of $300 \mathrm{~K}$. We attained a density of $1.02 \mathrm{~g} / \mathrm{cm}^{3}$ at the center of the channel, which agrees well with the value reported for channel widths of similar dimensions. ${ }^{3}$ After achieving the density, the system is equilibrated in the NVT ensemble at $300 \mathrm{~K}$ with a NoséHoover ${ }^{40,41}$ thermostat. To calculate the perpendicular component of the dielectric constant, an electric field of strength $E_{\text {ext }}=0.1 \mathrm{~V} / \AA$ is applied in the direction perpendicular to the walls. The total simulation time is $12 \mathrm{~ns}$. The first $6 \mathrm{~ns}$ is performed without an electric field and the rest with the field. A time step of $1 \mathrm{fs}$ is used throughout the simulation. Five independent simulations with different initial configurations are performed to calculate the statistical errors.

\section{RESULTS AND DISCUSSION}

Dielectric Response under Confinement. The static dielectric constant for a rigid nonpolarizable water model using 
Ewald's summation under conducting boundary conditions is given by $^{18}$

$$
\epsilon=1+\frac{4 \pi\left\langle M^{2}\right\rangle}{3 k_{\mathrm{B}} T V}
$$

where $\mathbf{M}(t)=\sum_{i=1}^{N} \boldsymbol{\mu}_{i}(t)$ is the total dipole moment of the system and $\mu_{i}$ is the dipole moment of the $i$ th molecule, and $k_{\mathrm{B}}$, $T$, and $V$ represent Boltzmann's constant, temperature, and volume of the system, respectively. We obtain the static dielectric constant of isotropic, homogeneous water as $72 \pm 3$, which is in good agreement with the available data in the literature. $^{19}$

Close to the confining walls, nonzero polarization exists even at a vanishing electric field. Hence, the net polarization density upon the application of an external field $E_{\text {ext }}$ is given by ${ }^{22}$

$$
\Delta \mathbf{P}=\langle\mathbf{P}\rangle_{E_{\text {ext }}}-\langle\mathbf{P}\rangle_{0}
$$

where $\langle\ldots\rangle_{E_{\text {ext }}}$ and $\langle\ldots\rangle_{0}$ denote the ensemble averages with and without the applied electric field, respectively. Due to the planar symmetry of the confined system in the $(x, y)$ plane, the average polarization has no components parallel to the surface (i.e., $\langle\mathbf{P}\rangle=(0,0, P(z)))$. Under constant displacement boundary conditions the net polarization density from eq 2 , relates to the inverse perpendicular dielectric response function $\left(\epsilon_{\perp}^{-1}(z)\right)$ as $^{22,23,42}$

$$
\epsilon_{\perp}^{-1}(z)=1-\frac{\Delta P(z)}{\epsilon_{0} E_{\mathrm{ext}}}
$$

where $E_{\text {ext }}$ is the external electric field applied perpendicular to the walls and $\epsilon_{0}$ is the vacuum dielectric permittivity.

The induced electric field due to the polarization of water in response to the external electric field $E_{\text {ext }}$ applied across $z$ is given by $^{43}$

$$
E_{\mathrm{p}}(z)=\frac{\int_{-\infty}^{z} \rho\left(z^{\prime}\right) \mathrm{d} z^{\prime}}{\epsilon_{0}}
$$

where $\rho\left(z^{\prime}\right)$ is the charge density distribution perpendicular to the surface. According to the multipole expansion approach the polarization field is related to the polarization density as ${ }^{23}$

$$
P(z)=-\epsilon_{0} E_{\mathrm{p}}(z)
$$

Therefore, from eqs 4 and 5 we get

$$
P(z)=-\int_{-\infty}^{z} \rho\left(z^{\prime}\right) \mathrm{d} z^{\prime}
$$

The local charge density $\rho(z)$ is defined as

$$
\rho(z)=\frac{1}{L_{x} L_{y}}\left\langle\sum_{i}^{N} q_{i} \delta\left(z-z_{i}\right)\right\rangle
$$

where $q_{i}$ is the charge of $i$ th atom and the ensemble average is replaced by a sample average in a bin, with $z$ as the mid-point of the slab/bin.

Figure $2 \mathrm{a}$ plots the net polarization density calculated from eqs 2 and 6 . We observe the net polarization density profile $(\Delta P)$ to be symmetric even though the polarization density profiles with $\left(P_{E_{\text {ext }}}\right)$ and without $\left(P_{0}\right)$ electric field are antisymmetric around the channel center. This behavior arises due to the alignment of water dipoles in the direction of the applied electric field (hydrogen atoms point toward the top wall and
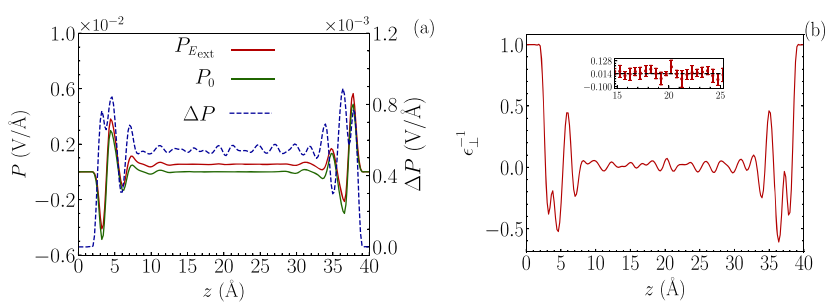

Figure 2. (a) Polarization density profile with (red) and without (green) an external field for water confined in a $40 \AA$ channel. The dashed line represents the variation of the net polarization density (due to $E_{\text {ext }}=0.1 \mathrm{~V} / \AA$ ) along the channel width. (b) Inverse dielectric response of water confined in the $40 \AA$ channel under an external perpendicular electric field of $E_{\text {ext }}=0.1 \mathrm{~V} / \AA$, calculated from eq 3 .

oxygen atoms point toward the bottom wall under the influence of an external field). Close to the walls, the perpendicular dielectric permittivity, given in Figure $2 b$, shows large amplitude variations similar to the net polarization density profile. However, from the inset plot of Figure $2 b$, we can observe that the dielectric response reaches the bulk value $\epsilon_{\perp}^{-1} \approx 1 / 71=0.014$ at the center of the channel matching the previous observations in the case of confined water. ${ }^{22,44}$

Similar to eq 2, the net electric field induced due to the polarization response under the external electric field $E_{\mathrm{ext}}$ is given by

$$
\Delta E_{\mathrm{p}}(z)=E_{\mathrm{p}, E_{\mathrm{ext}}}(z)-E_{\mathrm{p}, 0}(z)
$$

From Figure 3a, we observe that the magnitude of polarization field response $\Delta E_{\mathrm{p}}$ at the interfaces is much higher than the
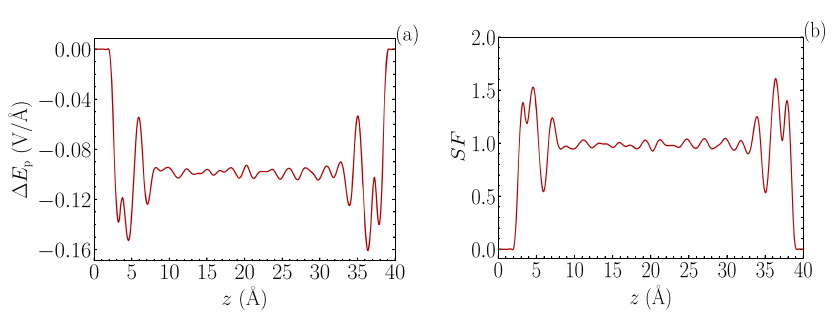

Figure 3. (a) Change in the polarization field and (b) variation of the screening factor, along the channel width under $E_{\text {ext }}=0.1 \mathrm{~V} / \AA$.

magnitude of the applied field. This results in the overscreening of the external field by the polarization field. The electric field screened by the polarization field can be measured using a dimensionless quantity called the screening factor defined as ${ }^{24}$

$$
\mathrm{SF} \equiv-\Delta E_{\mathrm{p}}(z) / E_{\mathrm{ext}}
$$

Using eqs 5 and 9, we can modify eq 3 for the perpendicular dielectric response $\epsilon_{\perp}^{-1}$ and write it in terms of the screening factor as

$$
\epsilon_{\perp}^{-1}(z)=1+\frac{\Delta E_{\mathrm{p}}(z)}{E_{\mathrm{ext}}}=1-\mathrm{SF}(z)
$$

From Figure $3 \mathrm{~b}$, we observe that SF > 1 close to the walls, affirming the overscreening phenomenon, which results in negative values of permittivity, as defined by eq 10 , for water near the graphene walls, and concurs with the previous observations on confined fluids. ${ }^{22-24,42}$ Close to the surface, the permittivity $1 / \epsilon_{\perp}^{-1}(z)$ diverges whenever the polarization field completely screens the external field, i.e., $\mathrm{SF}=1$, 
indicating the need of a nonlocal description of the dielectric response especially near the solid-water interfaces, as reported by Schaaf et al. ${ }^{45}$

The effective dielectric constant $\left(\epsilon_{\perp}^{\text {eff }}\right)$ at different regions in the channel is calculated from the formula given by Schlaich et al. $^{44}$

$$
\int_{L_{1}}^{L_{2}} \epsilon_{\perp}^{-1}(z) \mathrm{d} z=L_{\perp}^{\text {eff }}\left[\frac{1}{\epsilon_{\perp}^{\text {eff }}}-1\right]+L
$$

where $L_{\perp}^{\text {eff }}$ is the effective width of the region over which the effective dielectric constant $\epsilon_{\perp}^{\text {eff }}$ acts and $L=L_{2}-L_{1}$ is the distance enclosed by the region. The difference between the effective width and actual width of the region is

$$
\delta=L_{\perp}^{\text {eff }}-L
$$

As mentioned earlier (Figure $2 \mathrm{~b}$; inset plot), the center of the channel regains the bulk permittivity of water; hence, with $\epsilon_{\perp}^{\text {eff }}$ $=\epsilon_{\text {bulk }}$ for $L_{1}=15 \AA$ to $L_{2}=25 \AA$ and using eqs 11 and 12 , we can estimate the value of $\delta$. Now using this value of $\delta$ and eq 11 , we calculate the effective dielectric constant at different regions of water, which is given in Table 1 . We observe a

Table 1. Effective Out-of-Plane Dielectric Constant at Different Regions for a $40 \AA$ Channel $^{a}$

\begin{tabular}{ll}
\multicolumn{1}{c}{ region $(\AA)$} & \multicolumn{1}{c}{$\epsilon_{\perp}^{\text {eff }}$} \\
interface $[0-5$ and $35-40 \AA]$ & $5.099 \pm 0.443$ \\
bulk $[15-25 \AA]$ & 71 \\
total $[0-40 \AA]$ & $15.44 \pm 2.18$
\end{tabular}

${ }^{a}$ Values in square brackets indicate the distance enclosed by the respective region.

reduced effective dielectric permittivity for confined water, as reported in previous studies, ${ }^{9,28}$ with the least value of permittivity shown by the interface regions. The low dielectric constant at the interface indicates that the interfacial water molecules restrain themselves from aligning along the direction of the external field. We investigate the reason for this constrained alignment in the following sections.

Orientation of Water Molecules. Since a detailed analysis on the orientation of water molecules near planar surfaces has already been performed, ${ }^{29,46,47}$ we herein consider only the key orientational features that are relevant in the study of the anisotropic dielectric behavior of water under confinement. To re-examine the orientational preference of water molecules under confinement, we chose two different angles formed by a water molecule with the positive direction of the $z$-axis, viz., the angle $\phi_{\mu}$, formed by the molecular dipole moment of water $\mu_{i}$ and the $z$-axis, and the angle $\phi_{\mathrm{OH}}$, formed by the $\mathrm{OH}$ bonds of water and the $z$-axis.

Figure 4 shows the probability density distribution of the two angles defined above for the interfacial and bulk regions. Water dipoles and $\mathrm{OH}$ bonds in the bulk region have random orientation, whereas in the interfacial regions, they show preferential orientation. The peak at $\cos \phi_{\mu}=0$ indicates that molecular dipoles align parallel to the confining surfaces. The $\mathrm{OH}$ bond distribution shows the presence of two additional peaks at $\cos \phi_{\mathrm{OH}}=1$ and -1 besides the one at $\cos \phi_{\mathrm{OH}}=0$. This indicates that $\mathrm{OH}$ bonds have two preferred orientations: the first one, with $\mathrm{OH}$ bonds nearly parallel to the surface and the other with $\mathrm{OH}$ bonds parallel or antiparallel to the direction of $z$-axis.
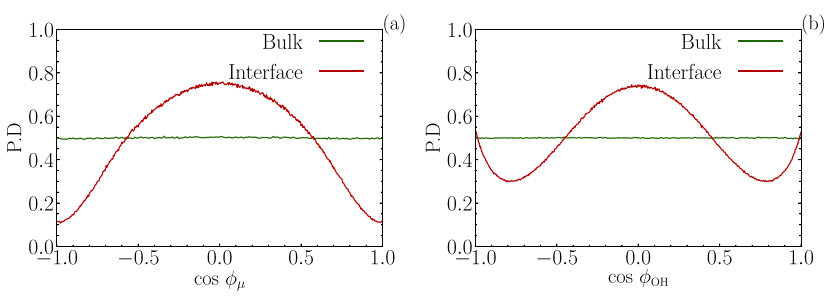

Figure 4. Probability distribution of angular orientation of (a) dipole moments and (b) OH bonds for bulk and interfacial regions.

To substantiate our observations, we also present the probability distribution of angles along the channel in Figure 5.
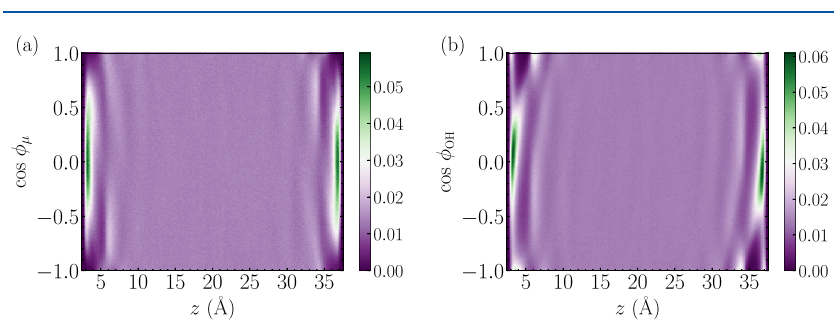

Figure 5. Probability distribution of angular orientation of (a) dipole moments and (b) $\mathrm{OH}$ bonds with respect to the distance from graphene walls.

As mentioned above, the molecular dipoles show preferential orientation only close to the surface (Figure 5a). The higher intensity of $\cos \phi_{\mathrm{OH}}=-1$ near the bottom graphene layer and $\cos \phi_{\mathrm{OH}}=1$ near the top graphene layer indicates presence of hydrogen atoms directly pointing to the confining walls. The normally oriented hydrogen atoms, as observed in our study, are a peculiar aspect of water-hydrophobic interactions. ${ }^{29}$

From the orientational analysis, we observe the interfacial water molecules to most likely orient along the planes parallel to the walls of the confinement, which results in a substantial restructuring of the hydrogen bond network at the interfaces. This preferential orientation of the water molecules is also the reason for the nonzero polarization observed (Figure 2a) close to the channel walls even at no external field, suggesting a spontaneous ferroelectric behavior for the interfacial water molecules. $^{48}$

Reorientation Times of Water Molecules under Confinement. We analyze the rotational and reorientational dynamics of water from the time correlation function $C_{l}$ defined as ${ }^{49}$

$$
C_{l}(t) \equiv\left\langle P_{l}\left(\mathbf{u}_{\mu}(t) \cdot \mathbf{u}_{\mu}(0)\right)\right\rangle
$$

where $P_{l}$ is the $l$ th Legendre polynomial and $\mathbf{u}_{\mu}$ is a unit vector parallel to the dipole moment of the molecule. Figure $6 a, b$ represents the results of $\ln \left(C_{l}(t)\right)$ for $l=1$ and 2 , at different regions of the confined water system.

The reorientational time $\tau_{l}$ is obtained from a linear fit of $\ln \left(C_{l}(t)\right)$ to

$$
y=-\frac{1}{\tau_{l}} x+b
$$

where $b$ is some constant. For isotropic diffusive motion, the rotational diffusion coefficient relates to the reorientation time as 49,50 

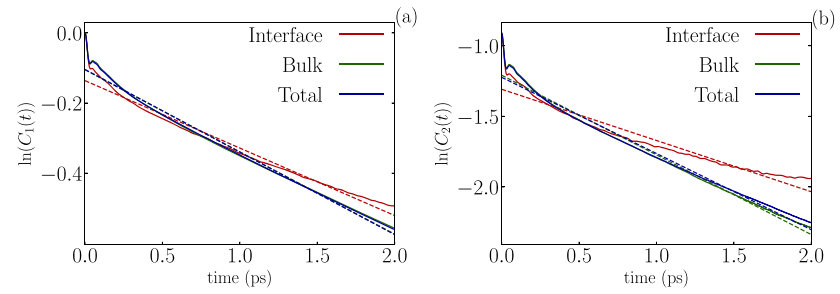

Figure 6. Logarithm of the time correlation functions when (a) $l=1$ and (b) $l=2$ for confined water. The dashed lines show the fitted curves to the logarithm of time correlation functions.

$$
D_{\mathrm{r}}=\frac{1}{l(l+1) \tau_{l}}
$$

The average values obtained for $\tau_{1}, \tau_{2}$, and $D_{\mathrm{r}}$ are provided in Table 2. For water in unconfined condition and the bulk region

Table 2. Correlation Times and Rotational Diffusion Coefficient for Confined and Unconfined Water

\begin{tabular}{llll}
\multicolumn{1}{c}{ region $(\AA)$} & \multicolumn{1}{c}{$\tau_{1}(\mathrm{ps})$} & \multicolumn{1}{c}{$\tau_{2}(\mathrm{ps})$} & $D_{\mathrm{r}}\left(\mathrm{ps}^{-1}\right)$ \\
interface & $5.2 \pm 0.7$ & $2.7 \pm 0.5$ & $0.06-0.09$ \\
bulk & $4.5 \pm 0.1$ & $1.8 \pm 0.1$ & $0.09-0.11$ \\
total & $4.4 \pm 0.1$ & $1.91 \pm 0.04$ & $0.09-0.11$ \\
liq. wat & $4.23 \pm 0.09$ & $1.78 \pm 0.03$ & $0.09-0.12$ \\
liq. wat (Expt) & $2-7.5$ & $1.7-2.6$ & \\
\hline
\end{tabular}

of the confined system, the values of reorientation times obtained are in close agreement with the reported values. ${ }^{51}$ This contrasts with the values close to the walls, where molecules reorient themselves on a longer timescale, consistent with the observations found for water near hydrophobic substrates. ${ }^{7,52-54}$ We conjecture this to arise from the anisotropic rotation of water molecules around the system axes near the surface. Since the rotational diffusion coefficient is inversely proportional to the reorientation time, $D_{\mathrm{r}}$ decreases as we move closer to the walls.

Now, we focus on the analysis of reorientation/rotation of water molecules along different axes. The correlation function $C_{l}^{\prime}$ along $x, y$, and $z$ axes is given by

$$
C_{l}^{\prime}(t) \equiv\left\langle P_{l}\left(\mathbf{u}_{\mu, \alpha}(t) \cdot \mathbf{u}_{\mu, \alpha}(0)\right)\right\rangle
$$

where $\mathbf{u}_{\mu, \alpha}$ is a unit vector parallel to the $\alpha$-component of the dipole moment of a molecule and $\alpha=x, y$, or $z$. Figure 7a,b shows $\ln \left(C_{1}^{\prime}(t)\right)$ along $x, y$, and $z$ axes for the bulk and interface, which is fitted to eq 14 to obtain the reorientation time.

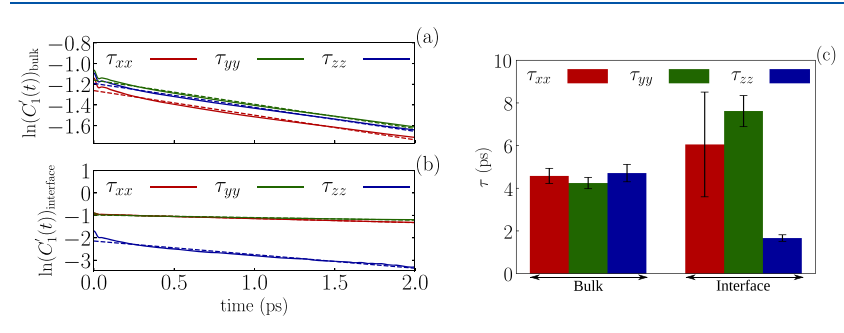

Figure 7. Logarithm of the time correlation functions along $x, y$, and $z$ axes of the system for (a) bulk and (b) interface regions. The dashed lines show the linear fit. (c) Values of reorientation time along different axes for water under confinement.
Figure $7 \mathrm{c}$ shows reorientation times along different axes for bulk and interfacial water molecules. In the bulk region, we observe that $\tau_{x x} \approx \tau_{y y} \approx \tau_{z z}$ whereas in the interfacial region, $\tau_{x x}$ $\approx \tau_{y y} \gg \tau_{z z}$. This means that in the interface water takes a much longer time to rotate out of the $x-y$ plane than doing a rotation in the $x-y$ plane. We also observe that $\tau_{x x \text {,interface, }}$ $\tau_{y y, \text { interface }}>\tau_{x x, \text { bulk }}, \tau_{y y, \text { bulk }}$ and $\tau_{z z \text {,interface }} \ll \tau_{z z \text {,bulk. }}$. This suggests that a significant suppression in the dipole rotation is exhibited in the direction perpendicular to the confining channel.

Orientation of Hydrogen Bonds under Confinement. Molecules are considered to be hydrogen-bonded if $r_{\mathrm{OO}^{\prime}}<3.5$ $\AA$ and $\theta_{\mathrm{HOO}^{\prime}}<30^{\circ}$, where $r_{\mathrm{OO}^{\prime}}$ is the distance between the donor and acceptor oxygen atoms, $\mathrm{O}$ and $\mathrm{O}^{\prime}$, and $\theta_{\mathrm{HOO}^{\prime}}$ is the angle between the $\mathrm{OH}$ bond and the $\mathrm{OO}^{\prime}$ vector. ${ }^{51,55,56}$ The orientation of hydrogen bonds is analyzed by measuring the angle made by the hydrogen bonds with the confinement axis. We here conjecture that the estimation of the planar hydrogen bonds formed by a water molecule can provide information on the restructuring of the hydrogen bond network under confinement. Figure 8 illustrates the criteria to consider a hydrogen bond as planar.

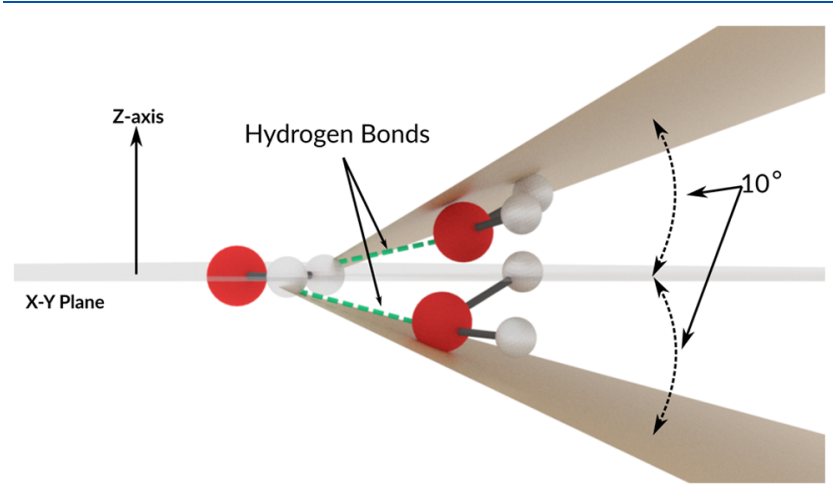

Figure 8. Schematic representation of the criteria to consider a planar hydrogen bond. A maximum of $10^{\circ}$ shift from the $x-y$ plane for the hydrogen bond is tolerated while considering the planarity of $\mathrm{H}$ bonds.

Figure 9a demonstrates the distribution of hydrogen bonds per molecule lying in planes parallel to the confining walls $(x-$
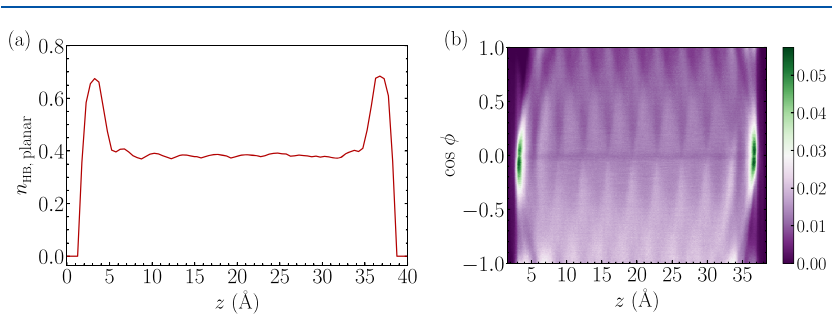

Figure 9. (a) Distribution of planar hydrogen bonds per molecule for water and (b) probability density distribution of angular orientation of hydrogen bonds confined in a $40 \AA$ channel. $\phi$ represents the angle between the hydrogen bond and $z$-axis.

$y$ plane). Planar hydrogen bonds restrict the rotation of water molecules away from the planes parallel to channel walls. The interface shows a higher density of planar hydrogen bonds compared with the bulk. Hence, the reorientation of water molecules becomes suppressed in the direction perpendicular to the planes parallel to the confining walls. This explains the 
longer time duration taken by the interfacial water molecules to rotate out of the $x-y$ plane of the system.

From Figure $9 \mathrm{~b}$, we find peaks at $\cos \phi=0$ at the interfaces, confirming our previous observation. Hence, we conclude that the polarization of the interfacial water molecules becomes difficult under the application of an electric field perpendicular to the channel walls.

The affinity of a system to undergo structural changes in response to external potential depends on the stability of the system structure. In other words, the highly stable layers undergo the least changes in their structure in response to an external field. Hence, this stability of the water layers is a critical factor that determines the ability of water molecules to reorient along the direction of an applied external electric field. Potential energy per molecule gives a qualitative measure in analyzing the stability of water layers as a function of distance from the surface. The potential energy per molecule at a layer is the average van der Waals interaction energy over all molecules present inside the layer and is given by

$$
\operatorname{PE}(z)=\sum_{j}^{N=\text { total no. of layers }} \frac{1}{n_{j}} \sum_{i}^{n_{j}}\left[P_{\mathrm{LJ}, \mathrm{WW}}^{i}+P_{\mathrm{LJ}, \mathrm{WC}}^{i}\right]
$$

where $n_{j}$ is the number of molecules in the $j$ th layer and $z$ is midpoint of layer. $P_{\mathrm{L} J \mathrm{wW}}^{i}$ and $P_{\mathrm{LJ}, \mathrm{WC}}^{i}$ are the interaction potential energy between the $i$ th water molecule and the other water molecules and graphene, respectively. From Figure 10a,

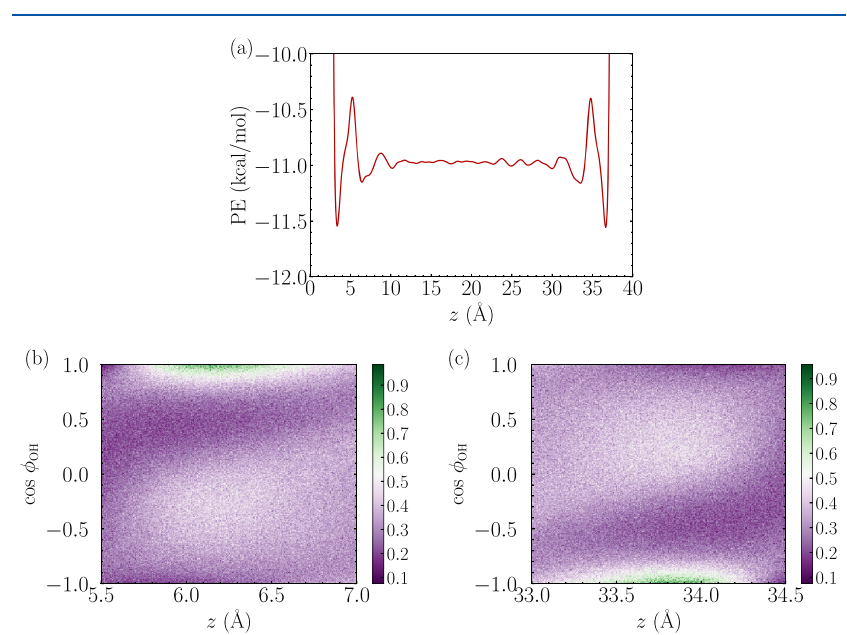

Figure 10. (a) Potential energy per molecule as a function of $z$ for confined water. Probability distribution of angular orientation of $\mathrm{OH}$ bonds at regions (b) 5.5-7.0 $\AA$ and (c) 33.0-34.5 $\AA$, inside the $40 \AA$ channel.

we observe a clear minimum in the potential energy per molecule at the interfacial regions $(0-5$ and $35-40 \AA)$. This stability close to the surface makes the realignment of the interfacial water molecules, under an external field, energetically relatively unfavorable. On the other hand, just adjacent to the interfaces (5.5-7.0 and 33.0-34.5 $\AA$ ), we see peaks in the potential energy profile. This corresponds to the $\mathrm{OH}$ bonds that point away from the planes parallel to the confining walls, as seen from Figure $10 \mathrm{~b}, \mathrm{c}$. The high potential energy per molecule at these layers indicates the labile nature of these "dangling" $\mathrm{OH}$ bonds due to their inability to form any significant hydrogen bonding.

\section{CONCLUSIONS}

In this work, we have investigated the physical origin of the reduced dielectric response of interfacial water, which is responsible for the extremely low perpendicular dielectric permittivity of confined water. We have shown that considering the orientation of hydrogen bonds under confinement is an effective way to rationalize this reduced response at the interfaces. At the interface, molecular dipoles orient parallel to the walls and $\mathrm{OH}$ bonds point either parallel or perpendicular to the confining walls. This indicates that water molecules prefer to stay parallel to the surface at the interface, giving rise to an increased concentration of planar hydrogen bonds near the channel walls. These planar hydrogen bonds restrict water from reorienting along the direction perpendicular to the atomic planes of the confining walls, resulting in the suppression of the dipole rotation in the perpendicular direction of the confining channel. Additionally, from the potential energy profile, we also observe an energetically favorable layer near the walls in comparison with bulk water. Hence, any disruption of the interfacial structure requires to overcome a significant energy barrier. This means, aligning the water molecules close to the surface under an electric field applied in the perpendicular direction of the channel becomes energetically demanding. The suppressed out-of-plane rotation due to the high density of planar hydrogen bonds coupled with the improved stability reduces the affinity of interfacial water molecules to align with an electric field applied perpendicular to the channel, which plays a significant role in the reduced perpendicular dielectric constant of water found at the solid-water interface.

Future investigation will address the possibility of a connection between hydrophobic forces ${ }^{57}$ and hydrogen bond dynamics. We observe the water in the interfacial regions to be much more energetically stable than in bulk even though the interfaces form a lesser number of hydrogen bonds compared with the bulk., ${ }^{3,58}$ In future research, we intend to focus on the energetics of the hydrogen bond structure near a hydrophobic surface. This may lead to further insight into the physical mechanism of hydrophobicity.

\section{AUTHOR INFORMATION}

\section{Corresponding Author}

*E-mail: sarith@iitm.ac.in.

ORCID

Sarith P. Sathian: 0000-0003-2756-7210

Notes

The authors declare no competing financial interest.

\section{ACKNOWLEDGMENTS}

The authors acknowledge the P. G. Senapathy Center for Computing Resources at IIT Madras for providing access to VIRGO supercluster. J.S.H. wishes to acknowledge Villum Foundation's Matter Grant (No. 16515).

\section{REFERENCES}

(1) Fayer, M. D.; Levinger, N. E. Analysis of water in confined geometries and at interfaces. Annu. Rev. Anal. Chem. 2010, 3, 89-107.

(2) Gun'ko, V.; Turov, V.; Bogatyrev, V.; Zarko, V.; Leboda, R.; Goncharuk, E.; Novza, A.; Turov, A.; Chuiko, A. Unusual properties of water at hydrophilic/hydrophobic interfaces. Adv. Colloid Interface Sci. 2005, 118, 125-172. 
(3) Cicero, G.; Grossman, J. C.; Schwegler, E.; Gygi, F.; Galli, G. Water confined in nanotubes and between graphene sheets: A first principle study. J. Am. Chem. Soc. 2008, 130, 1871-1878.

(4) De Luca, S.; Kannam, S. K.; Todd, B.; Frascoli, F.; Hansen, J. S.; Daivis, P. J. Effects of confinement on the dielectric response of water extends up to mesoscale dimensions. Langmuir 2016, 32, 4765-4773.

(5) Zhang, C.; Gygi, F.; Galli, G. Strongly anisotropic dielectric relaxation of water at the nanoscale. J. Phys. Chem. Lett. 2013, 4, 2477-2481.

(6) Senapati, S.; Chandra, A. Dielectric constant of water confined in a nanocavity. J. Phys. Chem. B 2001, 105, 5106-5109.

(7) Gekle, S.; Netz, R. R. Anisotropy in the dielectric spectrum of hydration water and its relation to water dynamics. J. Chem. Phys. 2012, 137, No. 104704.

(8) Itoh, H.; Sakuma, H. Dielectric constant of water as a function of separation in a slab geometry: a molecular dynamics study. J. Chem. Phys. 2015, 142, No. 184703.

(9) Fumagalli, L.; Esfandiar, A.; Fabregas, R.; Hu, S.; Ares, P.; Janardanan, A.; Yang, Q.; Radha, B.; Taniguchi, T.; Watanabe, K.; Gomila, G.; Novoselov, K.; Geim, A. Anomalously low dielectric constant of confined water. Science 2018, 360, 1339-1342.

(10) Meneses-Juárez, E.; Rivas-Silva, J. F.; González-Melchor, M. Static dielectric constant of water within a bilayer using recent water models: a molecular dynamics study. J. Phys.: Condens. Matter 2018, 30, No. 195001.

(11) Zhang, R.; Somasundaran, P. Advances in adsorption of surfactants and their mixtures at solid/solution interfaces. Adv. Colloid Interface Sci. 2006, 123-126, 213-229.

(12) Narayan, S.; Muldoon, J.; Finn, M.; Fokin, V. V.; Kolb, H. C.; Sharpless, K. B. "On water": Unique reactivity of organic compounds in aqueous suspension. Angew. Chem., Int. Ed. 2005, 44, 3275-3279.

(13) Bonthuis, D. J.; Horinek, D.; Bocquet, L.; Netz, R. R. Electrokinetics at aqueous interfaces without mobile charges. Langmuir 2010, 26, 12614-12625.

(14) De Luca, S.; Todd, B.; Hansen, J. S.; Daivis, P. J. Molecular dynamics study of nanoconfined water flow driven by rotating electric fields under realistic experimental conditions. Langmuir 2014, 30, 3095-3109.

(15) De Luca, S.; Todd, B.; Hansen, J.; Daivis, P. J. Electropumping of water with rotating electric fields. J. Chem. Phys. 2013, 138, No. 154712.

(16) Kirkwood, J. G. The dielectric polarization of polar liquids. J. Chem. Phys. 1939, 7, 911-919.

(17) Fröhlich, H. Theory of Dielectrics: Dielectric Constant and Dielectric Loss, 2nd ed.; O.U.P., 1958.

(18) Neumann, M. The dielectric constant of water. Computer simulations with the MCY potential. J. Chem. Phys. 1985, 82, 56635672.

(19) Rami Reddy, M.; Berkowitz, M. The dielectric constant of SPC/E water. Chem. Phys. Lett. 1989, 155, 173-176.

(20) Toney, M. F.; Howard, J. N.; Richer, J.; Borges, G. L.; Gordon, J. G.; Melroy, O. R.; Wiesler, D. G.; Yee, D.; Sorensen, L. B. Voltagedependent ordering of water molecules at an electrode-electrolyte interface. Nature 1994, 368, 444.

(21) Velasco-Velez, J.-J.; Wu, C. H.; Pascal, T. A.; Wan, L. F.; Guo, J.; Prendergast, D.; Salmeron, M. The structure of interfacial water on gold electrodes studied by $\mathrm{x}$-ray absorption spectroscopy. Science 2014, 346, No. 1259437.

(22) Ballenegger, V.; Hansen, J.-P. Dielectric permittivity profiles of confined polar fluids. J. Chem. Phys. 2005, 122, No. 114711.

(23) Bonthuis, D. J.; Gekle, S.; Netz, R. R. Profile of the static permittivity tensor of water at interfaces: Consequences for capacitance, hydration interaction and ion adsorption. Langmuir 2012, 28, 7679-7694.

(24) Parez, S.; Predota, M.; Machesky, M. Dielectric properties of water at rutile and graphite surfaces: effect of molecular structure. $J$. Phys. Chem. C 2014, 118, 4818-4834.
(25) Teschke, O.; Ceotto, G.; De Souza, E. Interfacial water dielectric-permittivity-profile measurements using atomic force microscopy. Phys. Rev. E 2001, 64, No. 011605.

(26) Schaaf, C.; Gekle, S. Dielectric response of the water hydration layer around spherical solutes. Phys. Rev. E 2015, 92, No. 032718.

(27) Sato, T.; Sasaki, T.; Ohnuki, J.; Umezawa, K.; Takano, M. Hydrophobic surface enhances electrostatic interaction in water. Phys. Rev. Lett. 2018, 121, No. 206002.

(28) Zhang, C. Note: On the dielectric constant of nanoconfined water. J. Chem. Phys. 2018, 148, No. 156101.

(29) Lee, C.-Y.; McCammon, J. A.; Rossky, P. The structure of liquid water at an extended hydrophobic surface. J. Chem. Phys. 1984, $80,4448-4455$.

(30) Galamba, N. Water's structure around hydrophobic solutes and the iceberg model. J. Phys. Chem. B 2013, 117, 2153-2159.

(31) Jahn, M.; Gekle, S. Bulk and interfacial liquid water as a transient network. Phys. Rev. E 2015, 92, No. 052130.

(32) Qi, W.; Zhao, H. Hydrogen bond network in the hydration layer of the water confined in nanotubes increasing the dielectric constant parallel along the nanotube axis. J. Chem. Phys. 2015, 143, No. 114708.

(33) Berendsen, H.; Grigera, J.; Straatsma, T. The missing term in effective pair potentials. J. Phys. Chem. 1987, 91, 6269-6271.

(34) Plimpton, S. Fast parallel algorithms for short-range molecular dynamics. J. Comput. Phys. 1995, 117, 1-19.

(35) Ryckaert, J.-P.; Ciccotti, G.; Berendsen, H. J. Numerical integration of the cartesian equations of motion of a system with constraints: molecular dynamics of n-alkanes. J. Comput. Phys. 1977, 23, 327-341.

(36) Badyal, Y.; Saboungi, M.-L.; Price, D.; Shastri, S.; Haeffner, D.; Soper, A. Electron distribution in water. J. Chem. Phys. 2000, 112, 9206-9208.

(37) Werder, T.; Walther, J. H.; Jaffe, R.; Halicioglu, T.; Koumoutsakos, P. On the water- carbon interaction for use in molecular dynamics simulations of graphite and carbon nanotubes. $J$. Phys. Chem. B 2003, 107, 1345-1352.

(38) Hockney, R. W.; Eastwood, J. W. Computer Simulation Using Particles, 2nd ed.; IOP: Bristol, U.K., 1988.

(39) Yeh, I.-C.; Berkowitz, M. L. Ewald summation for systems with slab geometry. J. Chem. Phys. 1999, 111, 3155-3162.

(40) Nosé, S. A unified formulation of the constant temperature molecular dynamics methods. J. Chem. Phys. 1984, 81, 511-519.

(41) Hoover, W. G. Canonical dynamics: equilibrium phase-space distributions. Phys. Rev. A 1985, 31, No. 1695.

(42) Bonthuis, D. J.; Gekle, S.; Netz, R. R. Dielectric profile of interfacial water and its effect on double-layer capacitance. Phys. Rev. Lett. 2011, 107, No. 166102.

(43) Glosli, J. N.; Philpott, M. R. Molecular dynamics study of interfacial electric fields. Electrochim. Acta 1996, 41, 2145-2158.

(44) Schlaich, A.; Knapp, E. W.; Netz, R. R. Water dielectric effects in planar confinement. Phys. Rev. Lett. 2016, 117, No. 048001.

(45) Schaaf, C.; Gekle, S. Spatially resolved dielectric constant of confined water and its connection to the non-local nature of bulk water. J. Chem. Phys. 2016, 145, No. 084901.

(46) Sedlmeier, F.; Janecek, J.; Sendner, C.; Bocquet, L.; Netz, R. R.; Horinek, D. Water at polar and nonpolar solid walls. Biointerphases 2008, 3, FC23-FC39.

(47) Stirnemann, G.; Rossky, P. J.; Hynes, J. T.; Laage, D. Water reorientation, hydrogen-bond dynamics and 2D-IR spectroscopy next to an extended hydrophobic surface. Faraday Discuss. 2010, 146, $263-281$.

(48) Gorshunov, B. P.; et al. Incipient ferroelectricity of water molecules confined to nano-channels of beryl. Nat. Commun. 2016, 7, No. 12842.

(49) Hansen, J.-P.; McDonald, I. Theory of Simple Liquids, 3rd ed.; Academic Press, 2006.

(50) Wong, V.; Case, D. A. Evaluating rotational diffusion from protein MD simulations. J. Phys. Chem. B 2008, 112, 6013-6024. 
(51) Laage, D.; Hynes, J. T. A molecular jump mechanism of water reorientation. Science 2006, 311, 832-835.

(52) Zichi, D. A.; Rossky, P. J. Solvent molecular dynamics in regions of hydrophobic hydration. J.Chem. Phys. 1986, 84, 28142822.

(53) Rocchi, C.; Bizzarri, A. R.; Cannistraro, S. Water dynamical anomalies evidenced by molecular-dynamics simulations at the solvent-protein interface. Phys. Rev. E 1998, 57, No. 3315.

(54) Choudhury, N. Orientational dynamics of water trapped between two nanoscopic hydrophobic solutes: A molecular dynamics simulation study. J. Chem. Phys. 2010, 133, No. 154515.

(55) Luzar, A.; Chandler, D. Hydrogen-bond kinetics in liquid water. Nature 1996, 379, 55.

(56) Rey, R.; Møller, K. B.; Hynes, J. T. Hydrogen bond dynamics in water and ultrafast infrared spectroscopy. J. Phys. Chem. A 2002, 106, 11993-11996.

(57) Donaldson, S. H., Jr.; Røyne, A.; Kristiansen, K.; Rapp, M. V.; Das, S.; Gebbie, M. A.; Lee, D. W.; Stock, P.; Valtiner, M.; Israelachvili, J. Developing a general interaction potential for hydrophobic and hydrophilic interactions. Langmuir 2015, 31, 2051-2064.

(58) Marti, J.; Nagy, G.; Gordillo, M.; Guardia, E. Molecular simulation of liquid water confined inside graphite channels: Thermodynamics and structural properties. J. Chem. Phys. 2006, 124, No. 094703. 